\title{
Hospitalist-Led Medicine Emergency Department Team: Associations With Throughput, Timeliness of Patient Care, and Satisfaction
}

\author{
Smitha R. Chadaga, MD ${ }^{1,3 *}$, Lee Shockley, MD, MBA ${ }^{2,3}$, Angela Keniston, MSPH ${ }^{1}$, Nancy E. Klock, MBA ${ }^{2}$, Susan Van Dyke, RN¹,
} Quin Davis, RN¹', Eugene S. Chu, MD'1,4

${ }^{1}$ Department of Medicine, Denver Health Medical Center, Denver, Colorado; '2Department of Emergency Medicine, Denver Health Medical Center, Denver, Colorado; ${ }^{3}$ University of Colorado School of Medicine Denver, Colorado; ${ }^{4}$ Division of Hospital Medicine, Department of Medicine, Boulder Community Hospital, Boulder, Colorado.

BACKGROUND: Admitted patients boarding in the emergency department (ED) leads to hospital diversion. Active bed management and care for boarded patients can improve throughput. We developed a hospital medicine ED (HMED) team to participate in active bed management, and to care for boarded patients, to decrease diversion and improve throughput.

METHODS: An HMED team was created to participate in active bed management and to care for boarded patients. The HMED team worked with the ED, nursing supervisors, and medical floors to manage inpatient beds. The primary outcome was percentage of hours of diversion attributed to lack of bed capacity. Secondary outcomes included the proportion of patients discharged within 8 hours of transfer to a medical floor, and the proportion of patients discharged from the ED. Promptness of clinical care was measured by rounding times. Satisfaction was obtained via survey.

RESULTS: There was a relative reduction of diversion due to medicine bed capacity of $27 \%(4.5 \%-3.3 \% ; P<0.01)$, a relative reduction in the percentage of patients transferred to a medicine floor and discharged within 8 hours of $67 \%$ (1.5\%-0.5\%; $P<0.01)$, and a relative increase in the number of discharges from the ED of admitted medicine patients of $61 \%(4.9 \%-7.9 \% ; P<0.001)$. Boarded admitted patients were rounded upon 2 hours earlier $(P<$ $0.0001)$ by the HMED team. Satisfaction with the HMED team was high.

CONCLUSION: An HMED team can improve patient flow and decrease ED diversion while providing more timely care to patients boarded in the ED. Journal of Hospital Medicine 2012;7:562-566. (C) 2012 Society of Hospital Medicine
Emergency department (ED) crowding leads to ambulance diversion, ${ }^{1}$ which can delay care and worsen outcomes, including mortality. ${ }^{2}$ A national survey showed that $90 \%$ of EDs were overcrowded, and $70 \%$ reported time on diversion. ${ }^{3}$ One of the causes of ED crowding is boarding of admitted patients. ${ }^{4}$ Boarding admitted patients decreases quality of care and satisfaction. ${ }^{5-7}$

Improved ED triage, bedside registration, physical expansion of hospitals, and regional ambulance programs have been implemented to decrease ED diversion. ${ }^{8-12}$ Despite these attempts, ED diversion continues to be prevalent.

Interventions involving hospitalists have been tested to improve throughput and quality of care for admitted medicine patients boarded in the ED. Howell and colleagues decreased ED diversion through active bed management by hospitalists. ${ }^{13}$ Briones and colleagues

*Address for correspondence and reprint requests: Smitha R. Chadaga, MD, Department of Medicine, Denver Health Medical Center, 777 Bannock, MC 4000, Denver, CO 80204-4507; Telephone: 303-436-6900; Fax: 303-436-7249; E-mail: smitha.chadaga@dhha.org Additional Supporting Information may be found in the online version of this article.

Received: December 5, 2011; Revised: May 19, 2012; Accepted: May 28, 2012

2012 Society of Hospital Medicine DOI 10.1002/jhm.1957

Published online in Wiley Online Library (Wileyonlinelibrary.com). dedicated a hospitalist team to patients boarded in the ED and improved their quality of care. ${ }^{14}$

Denver Health Medical Center (DHMC) is an urban, academic safety net hospital. In 2009, the ED saw an average of 133 patients daily and an average of 25 were admitted to the medical service. DHMC's ED diversion rate was a mean of $12.4 \%$ in 2009 . Boarded medicine patients occupied $16 \%$ of ED medicine bed capacity. Teaching and nonteaching medical floor teams cared for patients in the ED awaiting inpatient beds, who were the last to be seen. Nursing supervisors transferred boarded patients from the ED to hospital units. Patients with the greatest duration of time in the ED had priority for open beds.

ED diversion is costly. ${ }^{15,16}$ DHMC implemented codified diversion criteria, calling the administrator on-call prior to diversion, and increasing frequency of rounding in the ED, with no sustained effect seen in the rate of $\mathrm{ED}$ diversion.

In 2009, the DHMC Hospital Medicine Service addressed the issue of ED crowding, ED diversion, and care of boarded ED patients by creating a hospital medicine ED (HMED) team with 2 functions: (1) to provide ongoing care for medicine patients in the ED awaiting inpatient beds; and (2) to work with nursing supervisors to improve patient flow by adding physician clinical expertise to bed management. 


\section{METHODS}

\section{Setting and Design}

This study took place at DHMC, a 477-licensed-bed academic safety net hospital in Denver, Colorado. We used a pre-post design to assess measures of patient flow and timeliness of care. We surveyed ED attendings and nursing supervisors after the intervention to determine perceptions of the HMED team. This study was approved by the local institutional review board (IRB protocol number 09-0892).

\section{Intervention}

In 2009, DHMC, which uses Toyota Lean for quality improvement, performed a "Rapid Improvement Event" (RIE) to address ED diversion and care of admitted patients boarded in the ED. The RIE team consisted of hospital medicine physicians, ED physicians, social workers, and nurses. Over a 4-day period, the team examined the present state, created an ideal future state, devised a solution, and tested this solution.

Based upon the results of the RIE, DHMC implemented an HMED team to care for admitted patients boarded in the ED and assist in active bed management. The HMED team is a 24/7 service. During the day shift, the HMED team is composed of 1 dedicated attending and 1 allied health provider (AHP). Since the medicine services were already staffing existing patients in the ED, the 2.0 full-time equivalent (FTE) needed to staff the HMED team attending and the AHP was reallocated from existing FTE within the hospitalist division. During the evening and night shifts, the HMED team's responsibilities were rolled into existing hospitalist duties.

The HMED team provides clinical care for 2 groups of patients in the ED. The first group represents admitted patients who are still awaiting a medicine ward bed as of 7:00 AM. The HMED team provides ongoing care until discharge from the ED or transfer to a medicine floor. The second group of patients includes new admissions that need to stay in the ED due to a lack of available medicine floor beds. For

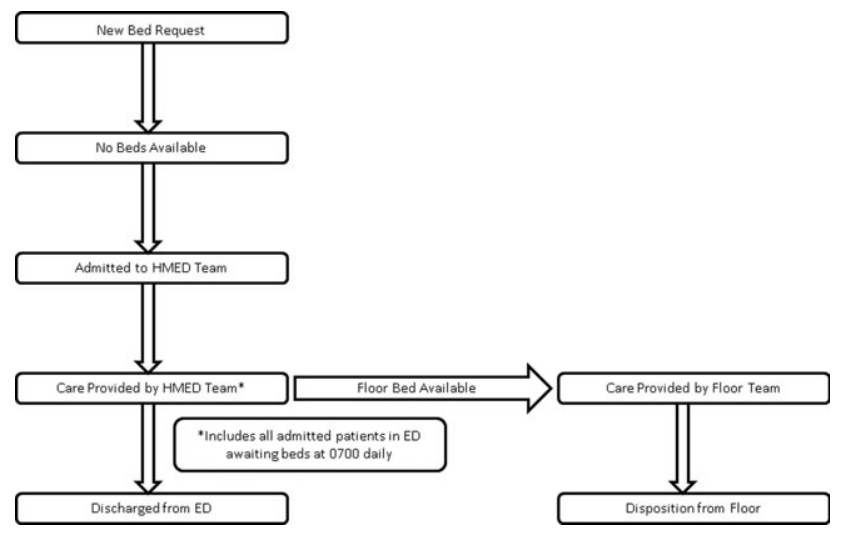

FIG. 1. Flow of care for patients boarded in the ED. Abbreviations: ED, emergency department; HMED, hospital medicine emergency department.

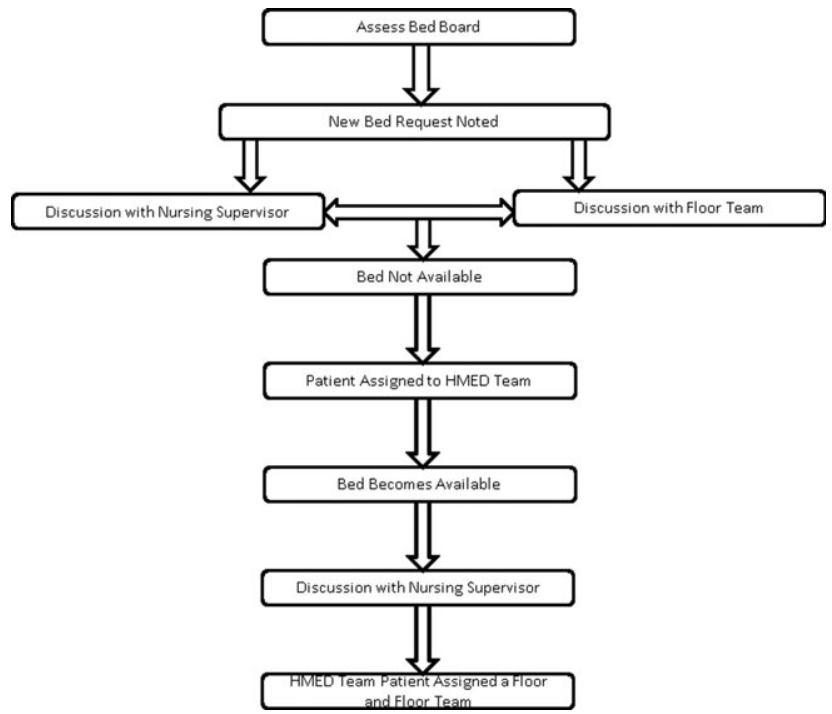

FIG. 2. Flow of active bed management by HMED team. Abbreviations: HMED, hospital medicine emergency department.

these patients, the HMED team initiates and continues care until discharge from the ED or transfer to a medical floor (Figure 1).

The physician on the HMED team assists nursing supervisors with bed management by providing detailed clinical knowledge, including proximity to discharge as well as updated information on telemetry and intensive care unit (ICU) appropriateness. The HMED team's physician maintains constant knowledge of hospital census via an electronic bed board, and communicates regularly with medical floors about anticipated discharges and transfers to understand the hospital's patient flow status (Figure 2).

The RIE that resulted in the HMED team was part of the Inpatient Medicine Value Stream, which had the overall goal of saving DHMC \$300,000 for 2009. Ten RIEs were planned for this value stream in 2009, with an average of $\$ 30,000$ of savings expected from each RIE.

\section{Determination of ED Diversion Time}

DHMC places responsibility for putting the hospital on an "ED Diversion" status in the hands of the Emergency Medicine Attending Physician. Diversion is categorized as either due to: (1) excessive ED volume for available ED beds-full or nearly full department, or full resuscitation rooms without the ability to release a room; or (2) excessive boarding-more than 12 admitted patients awaiting beds in the ED. Other reasons for diversion, such as acute, excessive resource utilization (multiple patients from a single event) and temporary limitation of resources (critical equipment becoming inoperative), are also infrequent causes of diversion that are recorded. The elapsed time during which the ED is on diversion status is recorded and reported as a percentage of the total time on a monthly basis. 


\section{Determination of ED Diversion Costs}

The cost of diversion at DHMC is calculated by multiplying the average number of ambulance drop-offs per hour times the number of diversion hours to determine the number of missed patients. The historical mean charges for each ambulance patient are used to determine total missed charge opportunity, which is then applied to the hospital realization rate to calculate missed revenue. In addition, the marginal costs related to Denver Health Medical Plan patients that were unable to be repatriated to DHMC from outlying hospitals, as a result of diversion, is added to the net missed revenue figure. This figure is then divided by the number of diversion hours for the year to determine the "cost" of each diversion hour. For 2009 , the cost of each hour of diversion at DHMC was $\$ 5000$.

\section{Statistical Analysis}

All analyses were performed using SAS Enterprise Guide 4.1 (SAS Institute, Inc, Cary, NC). A Student $t$ test or Wilcoxon rank sum test was used to compare continuous variables, and a chi-square test was used to compare categorical variables.

Our primary outcome was ED diversion due to hospital bed capacity. These data are recorded, maintained, and analyzed by a DHMC internally developed emergency medical services information system (EMeSIS) that interfaces with computerized laboratory reporting systems, and stores, in part, demographic data as well as real-time data related to the timing of patient encounters for all patients evaluated in the ED. To assess the effect of the intervention on ED diversion, the proportion of total hours on diversion due to medicine bed capacity was compared preimplementation and postimplementation with a chi-squared test.

Secondary outcomes for patient flow included: (1) the proportion of patients discharged within 8 hours of transfer to a medical floor; and (2) the proportion of admitted medicine patients discharged from the ED. These data were gathered from the Denver Health Data Warehouse which pools data from both administrative and clinical applications used in patient care. Chi-squared tests were also used to compare secondary outcomes preintervention and postintervention.

To measure the quality and safety of the HMED team, pre-ED and post-ED length of stay (LOS), 48-hour patient return rate, intensive care unit (ICU) transfer rate, and the total LOS for patients admitted to the HMED team and handed off to a medicine floor team were assessed with the Student $t$ test. To assess timeliness of clinical care provided to boarded medicine patients, self-reported rounding times were compared preintervention and postintervention with the Student $t$ test.

To assess satisfaction with the HMED team, an anonymous paper survey was administered to $\mathrm{ED}$ attendings and nursing supervisors 1 year after the intervention was introduced. The survey consisted of 5 questions,

\begin{tabular}{|c|c|c|c|}
\hline $\begin{array}{l}\text { Patients Admitted to } \\
\text { Medicine and Transferred } \\
\text { to a Medicine Floor }\end{array}$ & Pre & Post & $P$ Value \\
\hline NNo. & 1901 & 1828 & \\
\hline $\mathrm{Age}^{*}$ & $53 \pm 15$ & $54 \pm 14$ & 0.59 \\
\hline Gender ( $\%$ male) & $55 \%$ & $52 \%$ & 0.06 \\
\hline Race (\% white) & $40 \%$ & $34 \%$ & $<0.0001$ \\
\hline Insurance (\% insured) & $67 \%$ & $63 \%$ & 0.08 \\
\hline Charlson Comorbidity Index $†$ & $1.0[1.0,1.0]$ & $1.0[1.0,1.0]$ & 0.52 \\
\hline
\end{tabular}

Abbreviations: $\mathrm{Cl}$, confidence interval; HMED, hospital medicine emergency department; SD, standard deviation. ${ }^{*}$ Mean \pm SD. + Median $[95 \% \mathrm{Cl}]$.

and used a 5-point Likert scale ranging from strongly disagree (1) to strongly agree (5). Those answering agree or strongly agree were compared to those who were neutral, disagreed, or strongly disagreed.

\section{RESULTS}

The ED saw 48,595 patients during the intervention period (August 1, 2009-June 30,2010) which did not differ statistically from the 50,469 patients seen in the control period (August 1, 2008-June 30, 2009). The number of admissions to the medicine service during the control period (9727) and intervention period $(10,013)$, and the number of total medical/surgical admissions during the control $(20,716)$ and intervention $(20,574)$ periods did not statistically differ. ED staffing during the intervention did not change. The overall number of licensed beds did not increase during the study period. During the control period, staffed medical/surgical beds increased from 395 to 400 beds, while the number of staffed medical/surgical beds decreased from 400 to 397 beds during the intervention period. Patient characteristics were similar during the 2 time periods, with the exception of race (Table 1).

\section{Diversion Hours}

After implementation of the HMED team, there was a relative reduction of diversion due to medicine bed capacity of $27 \%(4.5 \%-3.3 \% ; P<0.01)$ (Table 2$)$. During the same time period, the relative proportion of hours on diversion due to ED capacity decreased by $55 \%(9.9 \%-5.4 \%)$.

\section{Bed Management and Patient Flow}

The HMED team rounded on boarded ED patients a mean of 2 hours and 9 minutes earlier (10:59 AM \pm $1: 09$ vs $8: 50 \mathrm{AM} \pm 1: 20 ; P<0.0001)$. After implementation of the HMED team, patients transferred to a medicine floor and discharged within 8 hours decreased relatively by $67 \%(1.5 \%-0.5 \% ; P<0.01)$, and discharges from the ED of admitted medicine patients increased relatively by $61 \%(4.9 \%-7.9 \% ; P<0.001)$ (Table 2). ED LOS, total LOS, 48-hour returns to the 


\begin{tabular}{|c|c|c|c|}
\hline \multicolumn{4}{|c|}{$\begin{array}{l}\text { TABLE 2. Comparison of the Proportion of Total } \\
\text { Hours on Divert Due to Bed Capacity, Discharges } \\
\text { Within } 8 \text { Hours of Being Admitted to a Medical Floor, } \\
\text { Length of Stay for Patients Rounded on by HMED } \\
\text { Team and Transferred to the Medical Floor, } \\
\text { Proportion of Admitted Medicine Patients } \\
\text { Discharged From the ED, ED Length of Stay for } \\
\text { Patients Cared for by the HMED Team, and 48-Hour } \\
\text { Return Rate and ICU Transfer Rate for Patients } \\
\text { Cared for by the HMED Team Preimplementation } \\
\text { and Postimplementation of the HMED Team }\end{array}$} \\
\hline & Pre & Post & $P$ Value \\
\hline $\begin{array}{l}\text { Divert hours due to bed } \\
\text { capacity (\%, hours) }\end{array}$ & $4.5 \%(3624)$ & $3.3 \%(3624)$ & 0.009 \\
\hline \multicolumn{4}{|c|}{ Admitted ED patients transferred to floor $†$} \\
\hline Discharged within $8 \mathrm{~h}(\%, \mathrm{~N}) \ddagger$ & $1.3 \%(1901)$ & $0.5 \%(1828)$ & 0.03 \\
\hline \multicolumn{4}{|c|}{ Boarded patients rounded on in the ED and transferred to the medical floor } \\
\hline Total length of stay (days, N)S & $2.6[2.4,3.2](154)$ & $2.5[2.4,2.6](364)$ & 0.21 \\
\hline \multicolumn{4}{|c|}{ All discharges and transfers to the floor $t$} \\
\hline Discharged from ED [\%, (N)] & $4.9 \%(2009)$ & $7.5 \%(1981)$ & $<0.001$ \\
\hline ED length of stay [hours, (N)]‡ & $12: 09 \pm 8: 44(2009)$ & $12: 48 \pm 10: 00(1981)$ & 0.46 \\
\hline Return to hospital < $48 \mathrm{~h}[\%$, (N)] & $4.6 \%(2009)$ & $4.8 \%(1981)$ & 0.75 \\
\hline Transfer to the ICU [\%, (N)] & $3.3 \%(2009)$ & $4.2 \%(1981)$ & 0.13 \\
\hline
\end{tabular}

Abbreviations: $\mathrm{Cl}$, confidence interval; DHMC, Denver Health Medical Center; ED, emergency department; HMED, hospital medicine emergency department; ICU, intensive care unit; SD, standard deviation. * January-May 2009 compared to January-May 2010. †August-December 2008 compared to August-December 2009. \pm Mean \pm SD. SMedian $[95 \% \mathrm{Cl}$.

ED, and ICU transfer rate for patients managed by the HMED team did not change (Table 2).

\section{Perception and Satisfaction}

Nine out of $15(60 \%)$ ED attendings and 7 out of 8 $(87 \%)$ nursing supervisors responded to the survey. The survey demonstrated that ED attendings and nursing supervisors believe the HMED team improves clinical care for boarded patients, communication, collegiality, and patient flow (Table 3).

\section{Financial}

The $27 \%$ relative reduction in ED diversion due to hospital bed capacity extrapolates to 105.1 hours a year of decreased diversion, accounting for $\$ 525,600$ of increased annual revenues.

\section{DISCUSSION}

This study suggests that an HMED team can decrease ED diversion, due to hospital bed capacity, by improving patient flow and timeliness of care for boarded medicine patients in the ED.

After participating in bed management, ED diversion due to a lack of medicine beds decreased. This is consistent with findings by Howell and colleagues who were able to improve throughput and decrease ED diversion with active bed management. ${ }^{13}$ Howell and colleagues decreased diversion hours due to temporary ED overload, and diversion hours due to a lack of telemetry or critical care beds. At DHMC, diversion is attributed to either a lack of ED capacity or lack of hospital beds. The primary outcome was the diversion rate due to lack of hospital beds, but it is possible that increased discharges directly from the ED contributed to the decrease in diversion due to $\mathrm{ED}$ capacity, underestimating the effect our intervention had on total ED diversion. There were no other initiatives to decrease diversion due to ED capacity during the study periods, and ED capacity and volume did not change during the intervention period.

While there were no statistically significant changes in staffed medical/surgical beds or medicine admissions, staffed medical/surgical beds during the intervention period decreased while there were more admissions to medicine. Both of these variables would increase diversion, resulting in an underestimation of the effect of the intervention.

Howell and colleagues improved throughput in the ED by implementing a service which provided active bed management without clinical responsibilities, ${ }^{13}$ while Briones and colleagues improved clinical care of patients boarded in the ED without affecting throughput. ${ }^{14}$ The HMED team improved throughput and decreased ED diversion while improving timeliness of care and perception of care quality for patients boarding in the ED.

By decreasing unnecessary transfers to medicine units and increasing discharges from the ED, patient flow was improved. While there was no difference in ED LOS, there was a trend towards decreased total LOS. A larger sample size or a longer period of observation would be necessary to determine if the trend toward decreased total LOS is statistically significant. ED LOS may not have been decreased because patients who would have been sent to the floor only to be discharged within 8 hours were kept in the ED to expedite testing and discharge, while sicker patients were sent to the medical floor. This decreased the turnover time of inpatient beds and allowed more boarded patients to be moved to floor units.

There was concern that an HMED team would fragment care, which would lead to an increased LOS for those patients who were transferred to a medical floor and cared for by an additional medicine team before discharge. ${ }^{17}$ As noted, there was a trend towards a decreased LOS for patients initially cared for by the HMED team.

In this intervention, hospital medicine physicians provided information regarding ongoing care of

TABLE 3. Survey Results of ED Attendings and Nursing Supervisors (\% Agree)

\begin{tabular}{lccc}
\hline $\begin{array}{l}\text { Postimplementation } \\
\text { of the HMED Team }\end{array}$ & $\begin{array}{c}\text { Total } \\
(\mathrm{n}=16)\end{array}$ & $\begin{array}{c}\text { ED Attendings } \\
(\mathrm{n}=9)\end{array}$ & $\begin{array}{c}\text { Nursing } \\
\text { Supervisors }(\mathrm{n}=7)\end{array}$ \\
\hline Quality of care has improved & 94 & 89 & 100 \\
Communication has improved & 94 & 89 & 100 \\
Collegiality and clinical decision-making & 94 & 100 & 89 \\
$\quad$ has improved & & & 100 \\
Patient flow has improved & 81 & 67 & 100 \\
HMED team is an asset to DHMC & 94 & 89 &
\end{tabular}

NOTE: Agree $=$ responded 4 or 5 on a 5 -point Likert scale. Abbreviations: DHMC, Denver Health Medical Center; ED, emergency department; HMED, hospital medicine emergency department. 
patients boarded in the ED to nursing supervisors. Prior to the intervention, nursing supervisors relied upon information from the ED staff and the boarded patient's time in the ED to assign a medical floor. However, ED staff was not providing care to boarded patients and did not know the most up-to-date status of the patient. This queuing process and lack of communication resulted in patients ready for discharge being transferred to floor beds and discharged within a few hours of transfer. The HMED team allowed nursing supervisors to have direct knowledge regarding clinical status, including telemetry and ICU criteria (similar to Howell and colleagues ${ }^{13}$ ), and readiness for discharge from the physician taking care of the patient.

By managing boarded patients, an HMED team can improve timeliness and coordination of care. Prior to the intervention, boarded ED patients were the last to be seen on rounds. The HMED team rounds only in the ED, expediting care and discharges. The increased proportion of boarded patients discharged from the ED by the HMED team is consistent with Briones and colleagues' clinically oriented team managing boarding patients in the ED. ${ }^{14}$

Potential adverse effects of our intervention included increased returns to the ED, increased ICU transfer rate, and decreased housestaff satisfaction. There was no increase in the 48-hour return rate and no increase in the ICU transfer rate for patients cared for by the HMED team. Housestaff at DHMC are satisfied with the HMED team, since the presence of the HMED team allows them to concentrate on patients on the medical floors.

This intervention provides DHMC with an additional $\$ 525,600$ in revenue annually. Since existing FTE were reallocated to create the HMED team, no additional FTE were required. In our facility, AHPs take on duties of housestaff. However, only 1 physician may be needed to staff an HMED team. This physician's clinical productivity is about $75 \%$ of other physicians; therefore, $25 \%$ of time is spent in bed management. At DHMC, other medicine teams picked up for the decreased clinical productivity of the HMED team, so the budget was neutral. However, using 2 FTE to staff 1 physician daily for 365 days a year, one would need to allocate 0.5 physician FTE (0.25 decrease in clinical productivity $\times 2$ FTE) for an HMED team.

Our study has several limitations. As a single center study, our findings may not extrapolate to other settings. The study used historical controls, therefore, undetected confounders may exist. We could not control for simultaneous changes in the hospital, however, we did not know of any other concurrent interventions aimed at decreasing ED diversion. Also, the decision to admit or not is partially based on individual ED attendings, which causes variability in practice. Finally, while we were able to measure rounding times as a process measure to reflect timeliness of care and staff perceptions of quality of care, due to our data infrastructure and the way our housestaff and attendings rotate, we were not able to assess more downstream measures of quality of care.

\section{CONCLUSION}

ED crowding decreases throughput and worsens clinical care; there are few proven solutions. This study demonstrates an intervention that reduced the percentage of patients transferred to a medicine floor and discharged within 8 hours, increased the number of discharges from the ED of admitted medicine patients, and decreased ED diversion while improving the timeliness of clinical care for patients boarded in the ED.

Disclosure: Nothing to report.

\section{References}

1. Fatovich DM, Nagree Y, Spirvulis P. Access block causes emergency department overcrowding and ambulance diversion in Perth, Western Australia. Emerg Med J. 2005;22:351-354.

2. Nicholl J, West J, Goodacre S, Tuner J. The relationship between distance to hospital and patient mortality in emergencies: an observational study. Emerg Med J. 2007;24:665-668.

3. Institute of Medicine. Committee on the Future of Emergency Care in the United States Health System. Hospital-Based Emergency Care: At the Breaking Point. Washington, DC: National Academies Press; 2007.

4. Hoot N, Aronsky D. Systematic review of emergency department crowding: causes, effects, and solutions. Ann Emerg Med. 2008;52: 126-136.

5. Pines JM, Hollander JE. Emergency department crowding is associated with poor care for patients with severe pain. Ann Emerg Med. 2008;51:1-5.

6. Pines JM, Hollander JE, Baxt WG, et al. The impact of emergency department crowding measures on time to antibiotics for patients with community-acquired pneumonia. Ann Emerg Med. 2007;50: 510-516.

7. Chaflin DB, Trzeciak S, Likourezos A, et al; for the DELAYED-ED Study Group. Impact of delayed transfer of critically ill patients from the emergency department to the intensive care unit. Crit Care Med. 2007;35:1477-1483.

8. Holroyd BR, Bullard MJ, Latoszek K, et al. Impact of a triage liaison physician on emergency department overcrowding and throughput: a randomized controlled trial. Acad Emerg Med. 2007;14:702-708.

9. Takakuwa KM, Shofer FS, Abuhl SB. Strategies for dealing with emergency department overcrowding: a one-year study on how bedside registration affects patient throughput times. Emerg Med J. 2007;32:337-342.

10. Han JH, Zhou C, France DJ, et al. The effect of emergency department expansion on emergency department overcrowding. Acad Emerg Med. 2007;14:338-343.

11. McConnell KJ, Richards CF, Daya M, Bernell SL, Weather CC, Lowe RA. Effect of increased ICU capacity on emergency department length of stay and ambulance diversion. Ann Emerg Med. 2005;5: 471-478.

12. Patel PB, Derlet RW, Vinson DR, Williams M, Wills J. Ambulance diversion reduction: the Sacramento solution. Am J Emerg Med. 2006;357:608-613.

13. Howell E, Bessman E, Kravat S, Kolodner K, Marshall R, Wright S. Active bed management by hospitalists and emergency department throughput. Ann Intern Med. 2008;149:804-810.

14. Briones A, Markoff B, Kathuria N, et al. A model of hospitalist role in the care of admitted patients in the emergency department. J Hosp Med. 2010;5:360-364.

15. McConnell KJ, Richards CF, Daya M, Weathers CC, Lowe RA. Ambulance diversion and lost hospital revenues. Ann Emerg Med. 2006; 48(6):702-710.

16. Falvo T, Grove L, Stachura R, Zirkin W. The financial impact of ambulance diversion and patient elopements. Acad Emerg Med. 2007; 14(1):58-62.

17. Epstein K, Juarez E, Epstein A, Loya K, Singer A. The impact of fragmentation of hospitalist care on length of stay. J. Hosp. Med. 2010;5: 335-338. 